\title{
Proteção contra corrosão da liga de alumínio 2024-T3 por filme de polipirrol eletrodepositado em ácido p-tolueno sulfônico
}

\section{Corrosion protection of aluminum alloy 2024-T3 by polypyrrole electrodeposited in p-toluenesulfonic acid}

Alex Fernandes de Souza ${ }^{1,3}$, Joares Lodovino Reis ${ }^{1}$, Liu Yao Cho², Barbara Ramos Ferreira², Andrea Santos Liu ${ }^{3}$

\section{RESUMO}

O polipirrol (PPy) é um polímero condutor que tem sido investigado para proteção de metais oxidáveis contra corrosão. Seu uso apresenta algumas vantagens, destacando-se baixa toxicidade, estabilidade química e facilidade de síntese química e eletroquímica. No presente trabalho, filmes de PPy foram eletrodepositados por cronoamperometria, em meio aquoso contendo ácido p-tolueno sulfônico. Os resultados dos ensaios de polarização potenciodinâmica, em meio agressivo de cloreto de sódio, mostraram que o potencial de corrosão foi deslocado para valores mais positivos quando o metal foi recoberto pelo PPy, indicando proteção anódica. Além disso, a Microscopia de Varredura Eletrônica de Varredura (MEV) indicou a formação de filmes de PPy compactos e homogêneos sobre a liga metálica, que não foram degradados, mesmo expostos por sete dias ao meio corrosivo contendo íons de cloreto.

Palavras-chave: Polipirrol; Alumínio 2024-T3; Eletrodeposição.

\section{ABSTRACT}

Polypyrrole (PPy) is a conducting polymer that has been investigated for corrosion protection of oxidizable metals. Its use presents some advantages as low toxicity, chemical stability and it can be synthetized by chemical and electrochemical methods. In this work, polypyrrole films were electrodeposited by chronoamperometry in p-toluene sulfonic acid. The results of potentiodynamic polarization in aggressive medium of chloride show that the corrosion potential was displaced to values that are more positive when the metal was coated by polypyrrole, indicating anodic protection. Furthermore, the SEM results indicate the formation of compact and homogeneous polypyrrole films on the metallic alloy.

Keywords: Polypyrrole; Aluminum 2024-T3; Electrodeposition.

'Departamento de Aeronáutica - Faculdade de Tecnologia de São José dos Campos - Prof. Jessen Vidal - São José dos Campos (SP) - Brasil ¿Universidade do Vale do Paraíba - Faculdade de Engenharia, Arquitetura e Urbanismo - Instituto de Pesquisa e desenvolvimento - São José dos Campos (SP) - Brasil

${ }^{3}$ Departamento de Química - Instituto Federal de Educação, Ciência e Tecnologia de São Paulo - São José dos Campos (SP) - Brasil.

Autor correspondente: Alex Fernandes de Souza - Faculdade de Tecnologia de São José dos Campos - Prof. Jessen Vidal - Av. Cesare Mansueto

Giulio Lattes, 1350 - CEP: 12247-014 - São José dos Campos (SP) - Brasil

Email: alex fernandes1989@live.com

Recebido: 26/02/2017 Aprovado: 23/03/2017 


\section{INTRODUÇÃO}

O alumínio e suas ligas têm uma ampla gama de aplicações, devido a sua baixa massa específica, elevada resistência à corrosão e alta condutividade elétrica ${ }^{(1)}$. Uma das principais finalidades da adição de elementos na liga de alumínio 2024-T3, principalmente o cobre, é o aumento da resistência mecânica, que se deve a formação de pequenos precipitados ${ }^{(2)}$. Entretanto, pode ocorrer a precipitação sob a forma de intermetálicos maiores que possuem um papel determinante na resistência à corrosão do material ${ }^{(3)}$.

Enquanto alguns elementos presentes na liga a enobrecem, como o manganês, outros a tornam mais suscetível à corrosão, como o cobre ${ }^{(4)}$, que após a solubilização, precipita a fase $\mathrm{CuAl}_{3}$ junto aos contornos de grão, causando o empobrecimento de cobre na região adjacente $^{(5)}$. A zona adjacente torna-se mais anódica em relação ao precipitado, onde a corrosão ocorre e se propaga ao longo dos contornos de grão, deteriorando esta zona mais empobrecida ${ }^{(6)}$.

Muitos inibidores de corrosão utilizados atualmente, a base de cromo hexavalente, são tóxicos e considerados cancerígenos e mutagênicos ${ }^{(7)}$.Desta forma, inúmeros grupos de pesquisa têm investigado alternativas ao uso do cromo hexavalente. Os filmes de polímeros condutores, como polianilina e polipirrol, são materiais com inúmeras aplicações no seu estado dopado ${ }^{(8,9)}$ ou parcialmente oxidado e que têm sido investigados para proteção de alumínio e suas ligas ${ }^{(10-12)}$. Estes polímeros são eletronicamente condutores, e podem ser denominados de revestimentos ativos, pois podem ocorrer interações eletrônicas, química e/ou eletroquímica com substratos metálicos ativos, como o alumínio ${ }^{(13)}$.

O polipirrol destaca-se pela estabilidade química, boa condutividade elétrica e facilidade de síntese química e eletroquímica. Seu monômero apresenta baixo potencial de oxidação e por isso, a síntese também pode ser realizada em meio aquoso ${ }^{(14,15)}$. Os filmes de PPy apresentam baixa rugosidade, flexibilidade, propriedades mecânicas boas e baixo custo de produção ${ }^{(16)}$. Todas essas características associadas à não toxicidade dos polímeros, torna-o vantajoso para ser utilizado como revestimento protetivo contra corrosão na liga de alumínio aeronáutico 2024-T3 $3^{(17-18)}$.

A polimerização eletroquímica direta de pirrol em eletrodos de metais ativos, como as ligas de alumínio, é complicada devido à oxidação do metal, que concorre com a oxidação do monômero, podendo inibir o crescimento do polímero ou reduzir a aderência do polímero ao metal ${ }^{(19,20)}$. Neste processo eletroquímico, a seleção do eletrólito é fundamental para evitar que o mesmo promova a oxidação do substrato metálico ${ }^{(21,22)}$. Estudos prévios, desenvolvidos em nossos laboratórios, mostraram que filmes homogêneos e compactos foram eletrodepositados sobre alumínio puro em meio de ácido p-tolueno sulfônico ${ }^{(11)}$.

Neste trabalho, foi investigada a eletrodeposição do PPy sobre a liga de alumínio 2024-T3, utilizando meio aquoso contendo ácido p-tolueno sulfônico. Através de ensaios eletroquímicos, também foi estudada a proteção contra corrosão da liga metálica pelo filme polimérico.

\section{MATERIAIS E MÉTODOS}

Os ensaios eletroquímicos foram realizados, utilizando-se um potenciostato modelo MQPG-01 da Microquímica. Os testes foram realizados em uma célula eletrolítica contendo três eletrodos: eletrodo de trabalho (liga Al 2024 - T3, embutida em teflon e com área exposta de $0,53 \mathrm{~cm}^{2}$ ); eletrodo de referência (Ag/AgCl); e eletrodo auxiliar (bastão de platina). A composição da liga de alumínio 2024 é apresentada na Tabela 1.

Tabela 1: Composição química \% (massa/massa) da liga de alumínio 2024.

\begin{tabular}{|c|c|c|c|c|c|c|}
\hline Elemento & $\mathrm{Fe}$ & $\mathrm{Cu}$ & Mn & Si & Sn & $\mathrm{Zn}$ \\
\hline Composição & 0,163 & 4,060 & 0,626 & 0,106 & $<0,183^{*}$ & $195,71^{*}$ \\
\hline
\end{tabular}

Antes dos ensaios eletroquímicos, as superfícies de alumínio foram desbastadas com lixas d'água com granulometria de 220 , 400, 600 e 1200 mesh, respectivamente. Em seguida, os eletrodos de alumínio foram enxaguados com água destilada.

A solução eletrolítica foi preparada dissolvendo-se $0,2 \mathrm{~mol} \mathrm{~L}^{-1}$ de ácido p-tolueno sulfônico e $0,2 \mathrm{~mol} \mathrm{~L}^{-1}$ de pirrol em água destilada.

A eletrodeposição do polipirrol (Ppy) foi realizada por voltametria cíclica, aplicando-se o potencial inicial de $-0,5 \mathrm{~V}$ até $+1,5 \mathrm{~V}$ em retorno a $-0,5 \mathrm{~V}$ vs $\mathrm{Ag} / \mathrm{AgCl}$, com velocidade de varredura de $20 \mathrm{mV} \mathrm{s}{ }^{-1}$, utilizando-se um Potenciostato/ Galvanostato modelo MQPG-01 da Microquímica, conectado e controlado por um microcomputador. Os filmes também foram eletrodepositados por cronoamperometria, aplicando-se potenciais de $0,8 \mathrm{~V}$ e $1,2 \mathrm{~V}$ vs. $\mathrm{Ag} / \mathrm{AgCl}$, durante 10 minutos.

A morfologia das superfícies revestidas com filmes de PPy foi analisada por Microscopia Eletrônica de Varredura (MEV), utilizando o equipamento Jeol JXA-840A.

A rugosidade dos filmes foi determinada por um rugosímetro, modelo TR100 - Surface Roughness Tester.

A proteção contra corrosão das superfícies de alumínio, recobertas pelos filmes de PPy, foi investigada por meio de curvas de polarização potenciodinâmica em uma solução de $\mathrm{NaCl} 0,1 \mathrm{~mol} \mathrm{~L}^{-1}$ $(\mathrm{pH}=6,4)$, a temperatura ambiente de $25^{\circ} \mathrm{C}$. Todas as amostras permaneceram em potencial de circuito aberto durante 4 horas. Os parâmetros de corrosão foram obtidos a partir das curvas de Tafel.

As superfícies de alumínio revestidas pelo filme de PPy também foram expostas ao meio corrosivo de cloreto por sete dias e, posteriormente, analisadas por MEV.

\section{RESULTADO E DISCUSSÃO}

Inicialmente, os filmes de polipirrol (Ppy) foram depositados por voltametria cíclica, variando o potencial entre $-0,5 \mathrm{~V}$ e $1,5 \mathrm{~V}$ a $20 \mathrm{mV} \mathrm{s}^{-1}$. Foi observado que as densidades de correntes anódicas, associadas com a formação do filme de PPy, aumentam com os sucessivos ciclos de varredura de potencial. Também pode ser 
notado que o valor do potencial, onde se inicia o crescimento do polímero, diminui com os sucessivos ciclos (Fig. 1).

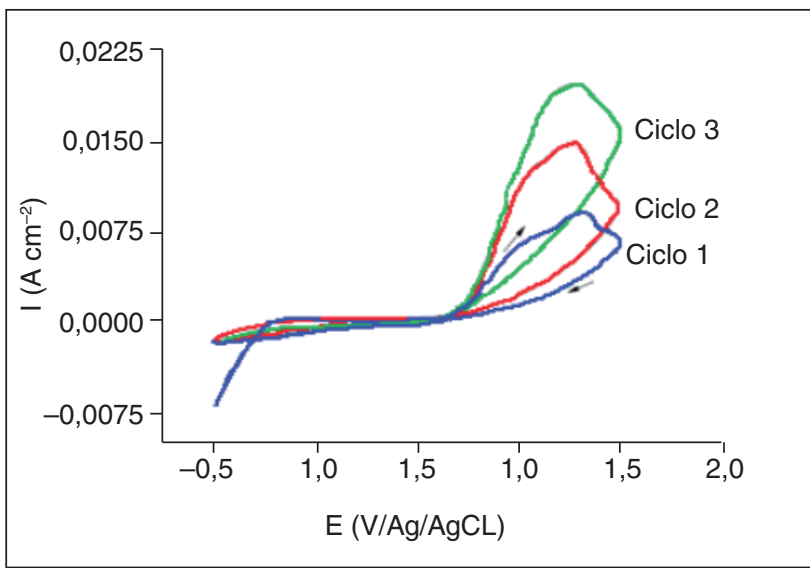

Figura 1: Voltametria cíclica para eletrodeposição do filme de PPy sobre a liga de alumínio 2024 - T3. Velocidade de varredura $=20 \mathrm{mV} \mathrm{s}^{-1}$

No voltamograma cíclico (ciclo 3), observa-se um pico anódico a 1,2 V, associado com a oxidação do monômero (pirrol), resultando na formação do polipirrol. Observou-se ainda que a carga total anódica aumentou com os sucessivos ciclos, sendo consistente com a maior quantidade de polímero formado no eletrodo ${ }^{23}$.

Posteriormente, foi investigada a eletrodeposição do filme de PPy sobre a superfície da liga de alumínio por cronoamperometria, aplicando potenciais de $0,8 \mathrm{~V}$ e $1,2 \mathrm{~V}$ durante 10 minutos. A Fig. 2 apresenta as curvas de corrente versus tempo obtidas nestes ensaios.

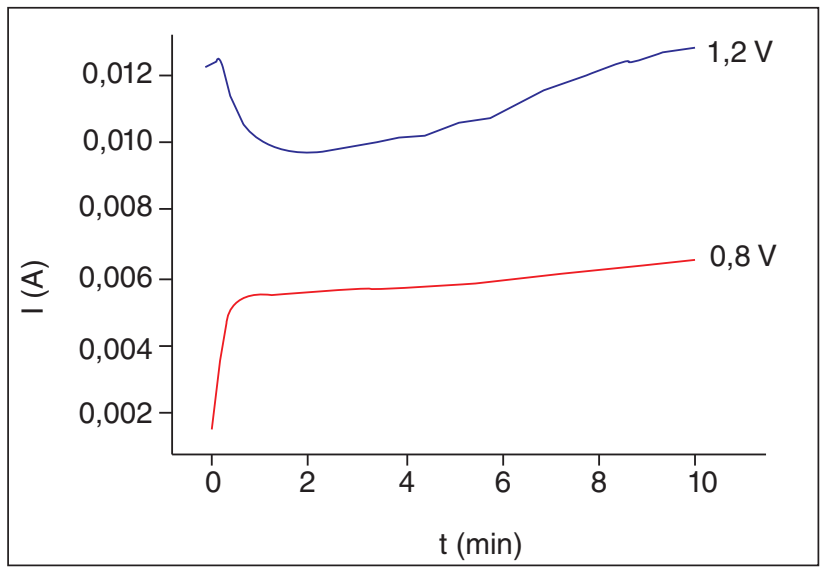

Figura 2: Curvas de potencial versus tempo para eletrodeposição do filme de PPy

Observou-se na Fig. 2 que após 2 minutos, ambas as correntes aumentaram, indicando o crescimento de filme de PPy, na forma condutora, que está sendo depositado na superfície do alumínio.

Ressalta-se ainda que a aplicação de potenciais inferiores a $0,8 \mathrm{~V}$ vs $\mathrm{Ag} / \mathrm{AgCl}$, o filme de PPy formado não recobriu completamente a superfície do eletrodo de alumínio.
Para analisar o desempenho dos filmes de PPy para proteger a liga de alumínio contra corrosão, foram realizados ensaios de polarização potenciodinâmica, em meio agressivo de cloreto de sódio $0,1 \mathrm{~mol} \mathrm{~L}^{-1}$. As curvas foram obtidas variando-se o potencial de $-1,0 \mathrm{~V}$ a $+0,5 \mathrm{~V}$ vs. $\mathrm{Ag} / \mathrm{AgCl}$, a velocidade de varredura de $1,0 \mathrm{mV} \mathrm{s}^{-1}$. Os parâmetros eletroquímicos, potenciais de corrosão e densidade de corrente de corrosão, foram obtidos, a partir das curvas de Tafel (Fig. 3).

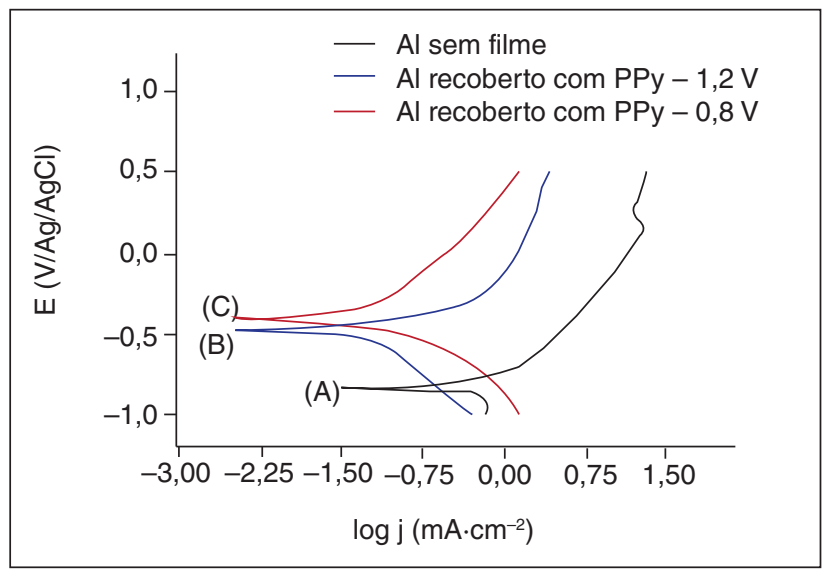

Figura 3: Curvas de Tafel para as superfícies de alumínio: (A) polida; (B) e (C) recobertas com filme de PPy.

Pode ser observado através das curvas de Tafel, que o potencial de corrosão para as superfícies de alumínio recobertas com os filmes de PPy é deslocado para a região mais positiva, indicando proteção anódica ${ }^{24}$.

Além disso, observou-se que as densidades de correntes anódicas, associadas com a dissolução do metal, são menores para as superfícies recobertas com o filme de PPy, indicando que o polímero pode inibir a oxidação do metal.

A eficiência do revestimento polimérico frente ao processo de corrosão foi obtida a partir da Eq. $1^{25}$.

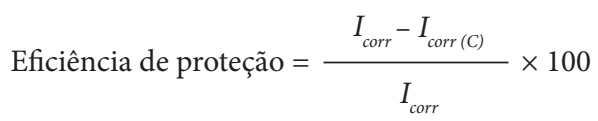

Onde $I_{\text {corr }}$ e $I_{\text {corr (c) }}$ são as densidades de corrente de corrosão na ausência e na presença do revestimento de PPy, respectivamente.

A Tabela 2 apresenta os parâmetros eletroquímicos (densidade de corrente de corrosão, eficiência de proteção e potenciais de corrosão), obtidos a partir das curvas de Tafel.

Ressalta-se que os valores de correntes anódicas, associadas a oxidação do metal, são menores para as superfícies de alumínio recobertas pelo filme de PPy. Por exemplo, considerando-se as densidades de corrente no potencial de $+0,25 \mathrm{~V}$, as correntes anódicas aumentam na sequência: superfície recoberta com PPy depositado a $0,8 \mathrm{~V}<$ superfície recoberta com PPy depositado a $1,2 \mathrm{~V}<$ alumínio sem filme. Estes resultados podem indicar que os filmes poliméricos atuam na proteção do alumínio contra corrosão. 
Tabela 2: Parâmetros eletroquímicos obtidos a partir das curvas de Tafel.

\begin{tabular}{|c|c|c|c|c|}
\hline Superfície de Alumínio 2024 & $\begin{array}{c}\text { Icorr } \\
\left(\mathrm{mA} \mathrm{cm}^{-2}\right)\end{array}$ & $\begin{array}{c}\text { Eficiência de proteção } \\
(\%)\end{array}$ & $\begin{array}{l}E_{\text {corr }} \\
(V)\end{array}$ & $\begin{array}{c}\text { I anódica a } 0,25 \mathrm{~V} \\
\left(\mathrm{~mA} \mathrm{~cm} \mathrm{~cm}^{-2}\right)\end{array}$ \\
\hline Sem recobrimento & 0,0316 & $\underline{-}$ & $-0,83$ & 15,13 \\
\hline Recoberta com PPy $(0,8 \mathrm{~V})$ & 0,0035 & $88,9 \%$ & $-0,41$ & 0,69 \\
\hline Recoberta com PPy $(1,2 \mathrm{~V})$ & 0,0036 & $88,6 \%$ & $-0,48$ & 1,86 \\
\hline
\end{tabular}

Os valores médios de rugosidade para as superfícies de alumínio recobertas com PPy são apresentados na Tabela 3.

Os resultados apresentados na Tabela 3 indicam que o filme de PPy é mais uniforme e menos rugoso, quando depositado, aplicando-se potenciais mais baixos $(0,8 \mathrm{~V}$ vs. $\mathrm{Ag} / \mathrm{AgCl})$.

Tabela 3: Valores médios de Rugosidade dos filmes de PPy.

\begin{tabular}{|c|c|}
\hline Superfície de Alumínio 2024 & Média $(\mu \mathrm{m})$ \\
\hline Recoberta com PPy $(0,8 \mathrm{~V})$ & 1,72 \\
\hline Recoberta com PPy $(1,2 \mathrm{~V})$ & 3,08 \\
\hline
\end{tabular}

Estudos apresentados na literatura indicaram que em potenciais mais elevados, o PPy pode sofrer superoxidação, influenciando na morfologia e no desempenho do polímero para proteger a superfície metálica contra corrosão ${ }^{26}$.

A morfologia da liga de alumínio recoberta com o filme de PPy foi analisada por Microscopia Eletrônica de Varredura (Fig. 4). Foi observado que a camada depositada é compacta e homogênea, o que justifica a proteção do metal contra corrosão, pois o filme dificulta a penetração de espécies corrosivas, como cloretos, inibindo a formação de pites no alumínio.

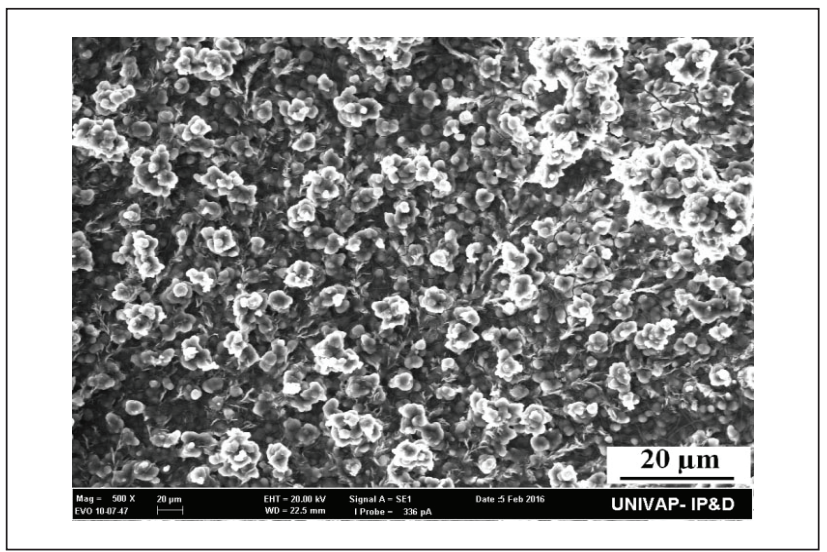

Figura 4: Microscopia Eletrônica de Varredura (MEV) da superfície do alumínio recoberta com filme de PPy a 0,8 V.

A micrografia indicou que o filme de PPy eletrodepositado recobriu uniformemente toda a superfície metálica. Este resultado está de acordo com estudos apresentados na literatura, onde os autores reportaram a formação de filmes de PPy homogêneos sem falhas ou defeitos, que inibiu a interação do metal com o meio corrosivo $^{27}$.
Foi realizado um ensaio de imersão em meio corrosivo de cloreto, com a superfície do alumínio recoberta com o filme de PPy depositado a $0,8 \mathrm{~V}$ vs. $\mathrm{Ag} / \mathrm{AgCl}$, durante 7 dias. Em seguida, a morfologia deste filme foi analisada por Microscopia Eletrônica de Varredura, conforme apresentado na Fig. 5.

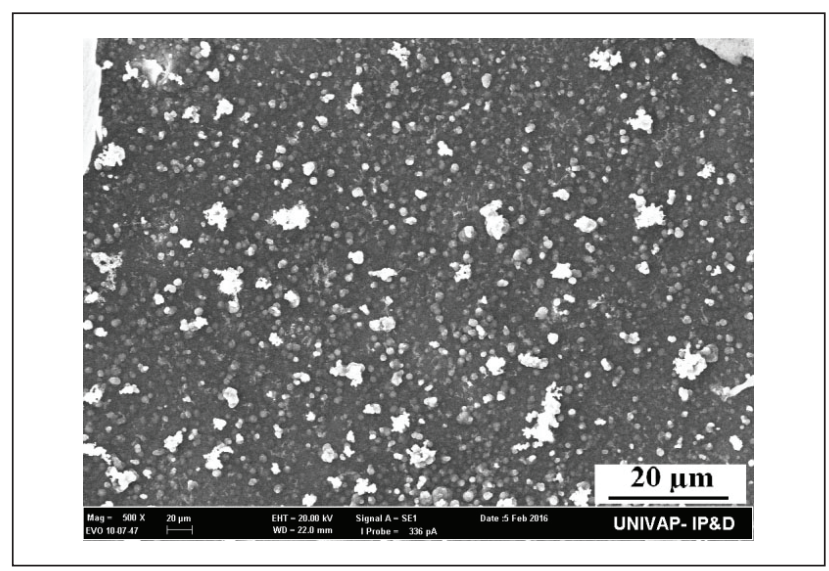

Figura 5: MEV da superfície do alumínio recoberto com PPy (depositado a 0,8 V), exposto ao meio corrosivo $0,1 \mathrm{~mol} \mathrm{~L}^{-1}$ de $\mathrm{NaCl}$.

A partir da análise por MEV realizada (Fig. 5), foi possível observar que o filme se manteve intacto após a imersão em meio

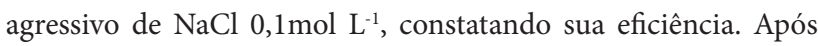
a remoção do filme polimérico foi observado que não houve a formação de pites na superfície do metal, confirmando a proteção e o bom desempenho do filme de PPy.

\section{CONCLUSÃO}

O estudo demonstrou que os filmes homogêneos e aderentes foram facilmente obtidos por cronoamperometria sobre a liga de alumínio 2024-T3. O teste de corrosão em solução salina mostrou que a interface PPy/liga metálica é bem estável, já que o filme permanece recobrindo toda a superfície, sem defeito, mesmo após sete dias, exposto ao meio corrosivo de cloreto. Portanto, conclui-se que as superfícies recobertas com as camadas poliméricas, enquanto estiverem íntegras, sem falhas, são menos susceptíveis à corrosão, em relação à liga de alumínio sem recobrimento, resultando em menores densidades de corrente de corrosão e deslocamento do potencial de corrosão para direções mais positivas.

\section{AGRADECIMENTOS}

Ao CNPq pelo auxílio concedido. 


\section{REFERENCES}

1. ZUH, D; WIM, J; OOIJ, V. Corrosion protection of AA 2024-T3 by bis-[3-(triethoxysilyl)propyl]tetrasulfide in neutral sodiumchloride solution. Part 1: corrosion of AA 2024-T3. Corrosion Science. 2003; 45: 2163-2175.

2. SHAO, M.; FU, Y.; HU, R.; LIN, C. A study on pitting corrosion of aluminum alloy 2024-T3 by scanning microreference electrode technique. Materials Science and Engineering. 2003; (A3) 44: 323-327.

3. CHEN, G.S.; GAO, M.; WEI, R.P. Microconstituent-Induced Pitting Corrosion in Aluminum Alloy 2024-T3. Corrosion Science. 1996; 52: (1): 8-15.

4. ZHAOA, J.; XIAA, L.; SEHGALB, A.; LUB, D.; MCCREERY, R. L. FRANKELB, G. S. Effects of chromate and chromate conversion coatings on corrosion of aluminum alloy 2024-T3. Surface and Coatings Technology. 2001; 140: 51-57.

5. HE, J. TAllman, D. E. BierWAGen, G. P. Conjugated Polymers for CorrosionControl: Scanning Vibrating Electrode Studies of Polypyrrole-Aluminum Alloy Interactions. Journal of Electrochemical Society. 2004; 151(12):644-651.

6. FERREIRA, C. A.; AEIVACH, S.; AARON, J. J.; LACAZET, P. C. Electrosynthesis of strongly adherent polypyrrole coatings on iron and mild steel in aqueous media. Electrochimica Acta. 1995; 41(11-12):1809-1996.

7. GELLING, V. J.; WIEST, M. M,; TALLMAN, D. E.; BIERWAGEN, G. P.; WALLACE, G. G. Electroactive-conducting polymers for corrosion control 4. Studies of poly(3-octyl pyrrole) and poly(3octadecyl pyrrole) on aluminum 2024-T3 alloy. Progress in Organic Coatings. 2011;43(1-3):149-157.

8. CONROY, K. G.; BRESLIN, C. B. The electrochemical deposition of polyaniline at pure aluminium: electrochemical activity and corrosion protection properties. Electrochimica Acta. 2003; 48: 721-732.

9. AKUNDY, G. S.; RAJAGOPALAN, R.; IROH, J. O. Electrochemical Deposition of Polyaniline-Polypyrrole Composite Coatings on Aluminum. Journal of Applied Polymer Science. 2002; 83: 19701977.

10. LIU, A. S.; OLIVEIRA, M. A. S. Electrodeposition of polypyrrole films on aluminum from tartrate aqueous solution. Journal of the Brazilian Chemical Society. 2007; 18(1): 143-152.

11 LIU, A. S.; BEZERRA, M. C.; CHO, L. Y. Electrodeposition of polypyrrole films on aluminum surfaces from a p-toluene sulfonic acid medium. Materials Research. 2009;12(4): 503-507.

12 JADHAV, N.; VETTER, C. A.; GELLING, V. J. The effect of polymer morphology on the performance of a corrosion inhibiting polypyrrole/aluminum flake composite pigment. Electrochimica Acta. 2013; 12: 28-43.

13 PALOUMPA, I.; YFANTIS, A.; HOFFMAN, P.; BURKOV, Y.; YFANTIS, D.; SCHMEIBER, D. Mechanisms to inhibit corrosion of Al alloys by polymeric conversion coatings. Surface and Coatings Technology. 2004; 180-181:308-312.

14 CASCALHEIRA, A. C.; AEIYACH, S. B.; LACAZE, P. C.; ABRANTES, L. M. Electrochemical synthesis and redox behaviour of polypyrrole coatings on copper in salicylate aqueous solution. Electrochimica Acta. 2003; 48: 2523-2529.

15 HERMELIN, E.; PETITJEAN, J.; AEIYACH, S.; LACROIX, J. C LACAZE, P. C. Industrial polypyrrole electrodeposition on zincelectroplated steel. Journal of Applied Eletrochemistry. 2001; 31 (905-911)

16 KIM, B. H.; PARK, D. H.; JOO, J.; YU, S. G.; LEE, S. H. Synthesis, characteristics, and field emission of doped and de-doped polypyrrole, polyaniline, poly(3,4-ethylenedioxythiophene) nanotubes and nanowires. Synthetic Metals. 2005; 150: 279-284.

17 MAIDEN, C. J.; MCMILLAN, A. R. An investigation of the protection afforded a spacecraft by a thin shiel. AIAA Journal. 1964 (2); 11 1992-1998.

18 LIU, A. S.; XAVIER, T. H. A.; CINTRA, E. P.; CHO, L. Y Electrodeposition of polypyrrole films on 2024 aluminum alloy in phosphoric acid solution. Materials Science Forum. 2014; (775776): 225-229.

19 ARENAS, M. A.; BAJOS, L. G.; DAMBORENEA, J. J.; OCÓN, P. Synthesis and electrochemical evaluation of polypyrrole coatings electrodeposited onto AA-2024 alloy. Progress in Organic Coatings. 2008; 62: 79-86.

20 IDLA, K.; INGANAS, B.; STRANDBERG, M. Good adhesion between chemically oxidised titanium and electrochemically deposited polypyrrole. Electrochimica Acta. 2000; 45: 2121 2130.

21 SHABANI-NOOSHABADI, M.; GHOREISHI, S. M.; BEHPOUR, M. Direct electrosynthesis of polyaniline-mont-morrilonite nanocomposite coatings on aluminum alloy 3004 and their corrosion protection performance. Corrosion Science. 2011; 53(9):3035-3042

22 MARTINS, N. C. T.; MOURA E SILVA, T.; MONTEMOR, M. F. FERNANDES, J. C. S.; FERREIRA, M. G. S. Electrodeposition and characterization of polypyrrole films on aluminium alloy 6061-T6. Electrochimica Acta. 2008; 53: 4754-4763.

23 FISHER, A. C. Electrode Dynamics. New York: Oxford Chemistry Primers, 1996. $84 \mathrm{p}$.

24 TALLMAN, D. E.; VANG, C.; WALLACE, G. G,; BIERWAGEN, G. P. Direct Electrodeposition of Polypyrrole on Aluminum and Aluminum Alloy by Electron Transfer Mediation. Journal of Electrochemical Society. 2002; 149(3):173-179.

25 KARPAGAM, V.; SATHIYANARAYANAN, S.; VENKATACHARI, G. Studies on corrosion protection of Al2024 T6 alloy by electropolymerized polyaniline coating. Current Applied Physics, 2008: 8: 93-98

26 MAZEIKIENE, R.; MALINAUSKAS, A. Kinetics of the electrochemical degradation of polypyrrole. Polymer Degradation and Stability. 2002: 75: 255-258.

27 LEHR, I.L.; SAIDMAN, S.B. Characterisation and corrosion protection properties of polypyrrole electropolymerised onto aluminium in the presence of molybdate and nitrate. Electrochimica Acta . 2006; 51: 3249-3255 